\title{
Synthesis of Exo-Methylenecyclopentane Derivatives via Radical Cyclization Starting from the Baylis-Hillman Adducts
}

\author{
Saravanan Gowrisankar, Hyun Seung Lee, and Jae Nyoung Kim* \\ Department of Chemistry and Institute of Basic Science, Chomam Kational Lhiversity, Gwangiu 500-757, Korea \\ E-mail: kiminachonnamackr \\ Received October 10, 2006
}

Key Words : Exo-methy lenecyclopentane. Radical ç̣clization. Baỵlis-Hillman adducts

Substituted cyclopentanes have been synthesized in a variety of ways $s^{i-3}$ including radical cyclizations of dienes or enynes ${ }^{2}$ and rhodium or palladium-catalyzed cyclization of enynes. ${ }^{2}$ These compounds also have been used as useful synthetic intermediates and act as an important backbone of some biologically important compounds. ${ }^{1.2}$

Recently, the synthesis of exo-ntethylenetetrahydrofurans was carried out via the $n-\mathrm{Bu}_{3} \mathrm{~S} \mathrm{H} \mathrm{H}$-mediated radical cyclization as the key step starting from the Baylis-Hillnan adduct by us and Shannugan's group. ${ }^{+}$Meanwhile we presumed that we could synthesize the corresponding carbon analog by applying similar strategy as shown in Scheme 1.

The reaction of the Baylis-Hillntan acetate and active methylene conpounds in the presence of $\mathrm{K}_{2} \mathrm{CO}_{3}$ afforded the starting material 1a-f in good yields as reported. Propargylation of $1 \mathbf{a}-\mathbf{f}$ under the influence of $\mathrm{NaH} / \mathrm{DMF} /$ propargyl bromide conditions gave 2a-f in $81-95 \%$ yields. With these compounds $2 \mathbf{a}-\mathbf{f}$ in our hand, we examined the radical cyclization. Tributyltinhydride-mediated radical cyclization of $2 \mathbf{a}-\mathbf{f}$ in benzene in the presence of AIBN produced cyclopentane derivatives 3 a-f selectively via the 5 exo-mig mode after hydrodestannylation. ${ }^{2.4}$ We could not observe the corresponding cyclohexane analogs, which could be formed via the 6-endo-rrig mode. as in our previous paper. ${ }^{4}$ The results are sunmiarized in Table 1 . It is interesting to note that we isolated only one stereoisomer in entries 5 and 6 . But. we did not determine the stereochemistry.

As a next trial. we examined the synthesis of exo-nethylene cyclohexane derivatives by using + as starting material.
The compound 4 was synthesized by using the DABCO salt concept (Scheme 2). which was already established in our group and used extensively for the regioselective introduction of nucleophile at the secondary position of the BaylisHilman adduct. However, the radical cyclization of 4 showed the formation of many intractable mixtures of products during the radical cyclization step.

When we subjected $\mathbf{3 a}$ under the Friedel-Crafts reaction conditions (Scheme 3), double bond isomerization occurred in high yield in short time to give 5 (rt. 1 h. $86 \%$ ) instead of the generally expected Friedel-Crafts reaction. The use of $\mathrm{AlCl}_{3}$ instead of sulfuric acid showed no reaction.

In summary. we disclosed the facile synthesis of highly substituted cyclopentane derivatives from the modified Baylis-Hillman adducts by radical cyclization protocol.

\section{Experimental Section}

Typical procedure for the synthesis of 2a: To a stirred solution of $1 \mathrm{a}^{5}$ (306 $\mathrm{mg}, 1.0 \mathrm{mmol}$ ) in DMF (2 mL) was added $\mathrm{NaH}(60 \%$ in mineral oil, $48 \mathrm{mg}, 1.2 \mathrm{mmol})$. To the reaction mixture propargyl bromide ( $179 \mathrm{mg} .1 .2 \mathrm{mmol}$ ) was added and the reaction mixture was stirred at room temperature for $5 \mathrm{~h}$. The reaction mixture was poured into cold $\mathrm{NH}_{4} \mathrm{Cl}$ solution and extracted with ether. After the usual aqueous extractive workup with ether and column chromatographic purification process (hexanes/EtOAc. 7 : 3) we obtained 2 a (310 $\mathrm{ng}, 90 \%$ ) as colorless oil. Other compounds 2b-f were synthesized analogously and the spectroscopic

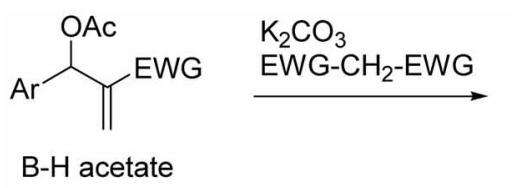

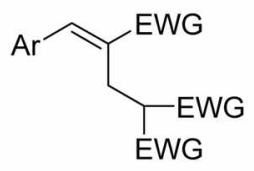

1

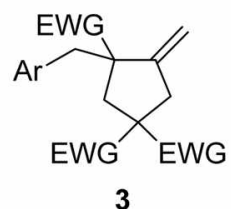

3 propargyl bromide $\mathrm{NaH}$, DMF

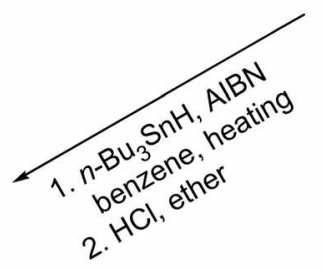

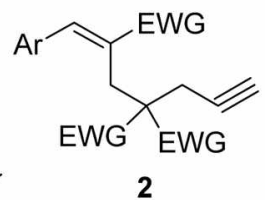

2

Scheme 1 
Table 1. Synthesis of methylenecyclopentane derivatives

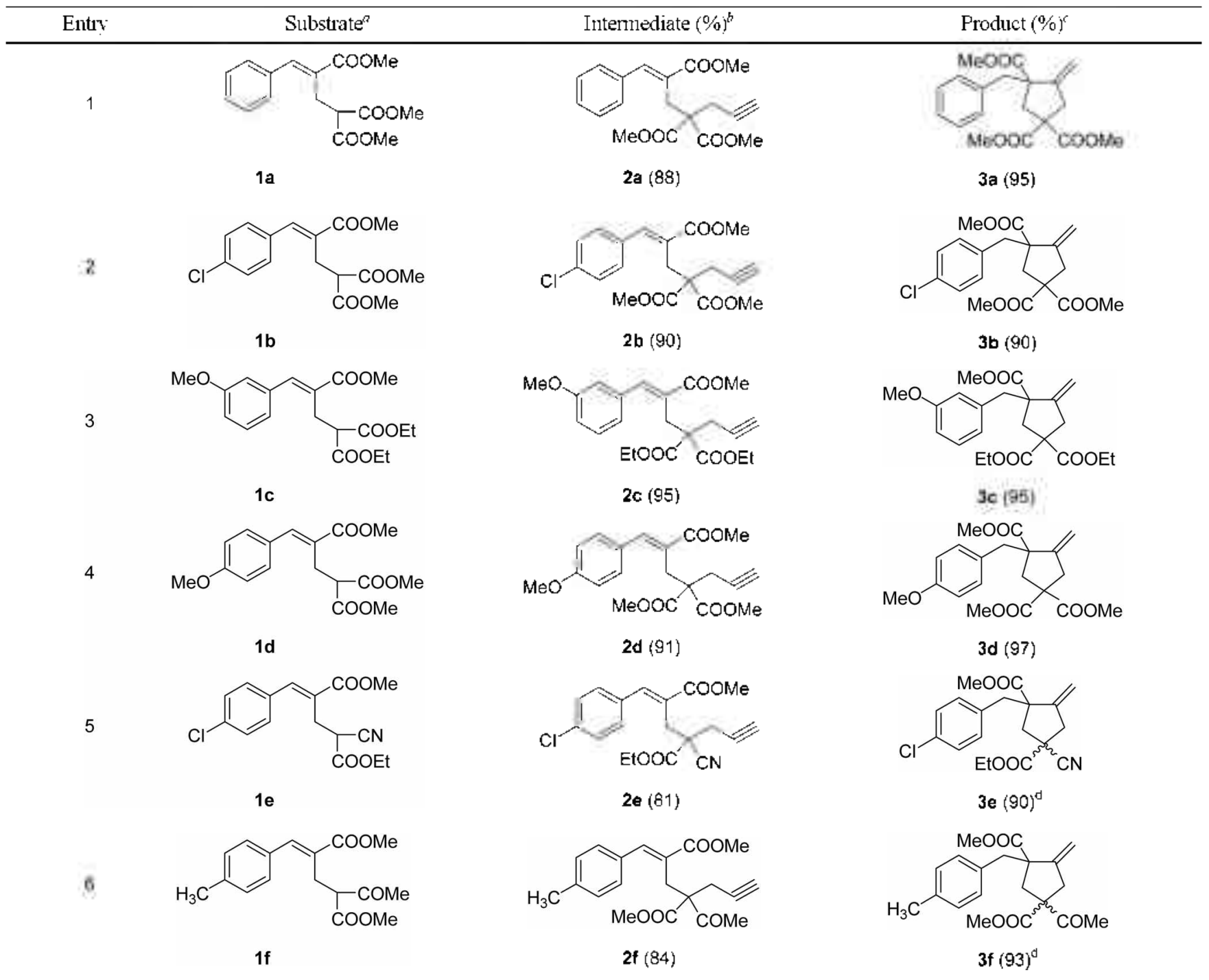

"Starting materials 1a-f were prepared from the reaction of the corresponding Baylis-Hillman acetates and active methylene compounds according to the reported method. "Conditions: Substrate 1 (1.0 mmol), NaH (1.2 equiv). DMF, propargyl bromide ( 1.2 equiv). rt. $12 \mathrm{~h}$. "Conditions: (i) Intermediate 2 ( 1.0 equiv), $n-\mathrm{Bu}_{3} \mathrm{SnH}$ (1.1 equiv), $\mathrm{AlBN}$ (cat). benzene. reflux $1 \mathrm{~h}$ and (ii) conc $\mathrm{HCl}$ ( 3 drops). ether. $\mathrm{rt}$. $\mathrm{l} \mathrm{l}$. "We obtained only ane isomer but we did not determine the stereochemistry.

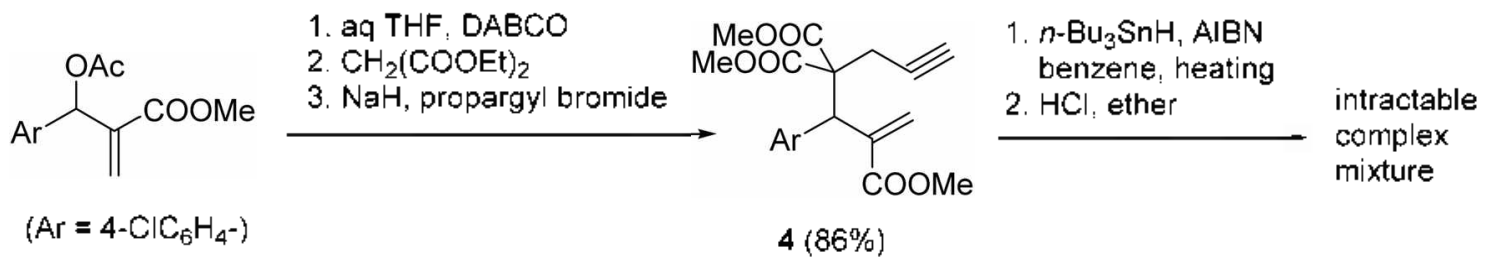

Scheme 2

3a $\underset{0^{\circ} \mathrm{C}-\mathrm{rt}, 1 \mathrm{~h}}{\stackrel{\mathrm{H}_{2} \mathrm{SO}_{4} \text { (3.0 equiv), } \mathrm{PhH}}{\longrightarrow}}$

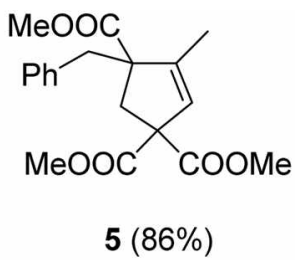

Scheme 3 data of $2 a-f$ and 4 are as follows.

Compound 2a: colorless oil: 88\%: IR (neat) 3290, 2952 . 1736. $1437.1244 \mathrm{~cm}^{-1}$; ${ }^{1} \mathrm{H}$ NMR (CDCl $\left.3.300 \mathrm{MHz}\right) \delta 1.54$ (t. $J=2.7 \mathrm{~Hz}, 1 \mathrm{H}), 2.63$ (d. $J=2.7 \mathrm{~Hz} .2 \mathrm{H}), 3.52(\mathrm{~s}, 2 \mathrm{H})$. 3.62 (s. 6H). 3.77 (s. 3H). 7.26-7.42 (m. 5H). 7.83 (s. 1H): ${ }^{13} \mathrm{C}$ NMR $\left(\mathrm{CDCl}_{3} .75 \mathrm{MHz}\right) \delta 22.98,28.16 .52 .02 .52 .62$ $(2 \mathrm{C}), 57.13,70.70 .78 .42,128.04,128.09,128.28,128.45$, $129.21,135.21,143.18,168.40,170.17$. 
Compound 2b: colorless oil; 90\%; IR (neat) 3302, 2954. 2256. 1732. 1435. $1244 \mathrm{~cm}^{-1}$. ${ }^{1} \mathrm{H} \mathrm{NMR}\left(\mathrm{CDCl}_{3}, 300 \mathrm{MHz}\right) \delta$ 1.57 (t. $J=2.7 \mathrm{~Hz}, \mathrm{IH}) .2 .62$ (d. $J=2.7 \mathrm{~Hz}, 2 \mathrm{H}) .3 .48$ (s. $2 \mathrm{H}$ ). 3.65 (s. $6 \mathrm{H}$ ) 3.77 (s. $3 \mathrm{H}$ ) 7.34 (s. $4 \mathrm{H}$ ). 7.77 (s. $1 \mathrm{H}$ )

Compound 2e: colorless oil: 95\%: IR (neat) 3284, 2983. 1732. $1435.1242 \mathrm{~cm}^{-1}$ : ${ }^{1} \mathrm{H}$ NMR $\left(\mathrm{CDCl}_{3}, 300 \mathrm{MHz}\right) \delta 1.15-$ $1.22(\mathrm{~m} .6 \mathrm{H}) .1 .63(\mathrm{t} . J=2.7 \mathrm{~Hz}, \mathrm{lH}) .2 .67(\mathrm{~d} . J=2.7 \mathrm{~Hz}$. $2 \mathrm{H}$ ). 3.53 (s. $2 \mathrm{H}$ ). 3.76 (s. $3 \mathrm{H}$ ). 3.80 (s. $3 \mathrm{H}$ ). $3.97-4.23$ (m. $4 \mathrm{H}), 6.94-7.00(\mathrm{~m}, 3 \mathrm{H}), 7.24-7.29(\mathrm{~m}, \mathrm{lH}) .7 .77(\mathrm{~s} .1 \mathrm{H})$.

Compound 2d: colorless oil: 91\%: IR (neat) 3288. 2954. 1736. 1606. 1512. $1255 \mathrm{~cm}^{-1}$, ${ }^{1} \mathrm{H} \mathrm{NMR}\left(\mathrm{CDCl}_{3}, 300 \mathrm{MHz}\right) \delta$ $1.65(\mathrm{t} . J=2.7 \mathrm{~Hz}, 1 \mathrm{H}) .2 .67(\mathrm{~d} . J=2.7 \mathrm{~Hz}, 2 \mathrm{H}) .3 .55(\mathrm{~s}$. $2 \mathrm{H}$ ). 3.64 (s. $6 \mathrm{H}$ ). 3.76 (s. $3 \mathrm{H}$ ). 3.82 (s. $3 \mathrm{H}$ ). 6.88 (d. $J=8.7$ $\mathrm{Hz}, 2 \mathrm{H}) .7 .41$ (d. $J=8.7 \mathrm{~Hz}, 2 \mathrm{H}) .7 .77$ (s. $1 \mathrm{H}$ )

Compound 2e: colorless oil: 81\%: IR (neat) 3294. 2985. 2952. 2251. 1747. $1259 \mathrm{~cm}^{-1}$; ${ }^{1} \mathrm{H} \mathrm{NMR}\left(\mathrm{CDCl}_{3}, 300 \mathrm{MHz}\right) \delta$ $1.30(\mathrm{t} . J=7.2 \mathrm{~Hz} .3 \mathrm{H}$ ). 2.10 (t. $J=2.7 \mathrm{~Hz} .1 \mathrm{H}$ ). 2.68 (dd. $J$ $=16.5$ and $3.0 \mathrm{~Hz}, 1 \mathrm{H}) .2 .79(\mathrm{dd} . J=16.5$ and $3.0 \mathrm{~Hz}, 1 \mathrm{H})$. 3.22 (d. $J=14.1 \mathrm{~Hz} .1 \mathrm{H}$ ). 3.32 (d. $J=14.1 \mathrm{~Hz} .1 \mathrm{H}$ ). 3.82 (s. $3 \mathrm{H}) .4 .12-4.27(\mathrm{~m} .2 \mathrm{H}) .7 .32-7.40(\mathrm{~m} .4 \mathrm{H}) .7 .91(\mathrm{~s} . \mathrm{lH})$.

Compound 2f: colorless oil: 84\%: IR (neat) 3284. 2954. 1736. 1437. $1242 \mathrm{~cm}^{-1} ;{ }^{1} \mathrm{H} \mathrm{NMR}\left(\mathrm{CDCl}_{3}, 300 \mathrm{MHz}\right) \delta 1.53$ (t. $J=2.7 \mathrm{~Hz} .1 \mathrm{H}$ ). 2.14 (s. $3 \mathrm{H}$ ). 2.34 (s. $3 \mathrm{H}$ ). 2.55 (d. $J=2.7$ Hz. $1 \mathrm{H}) .3 .38(\mathrm{~d} . J=14.7 \mathrm{~Hz}, 1 \mathrm{H}) .3 .54(\mathrm{~d} . J=14.7 \mathrm{~Hz}, 1 \mathrm{H})$. 3.64 (s. $3 \mathrm{H}) .3 .74$ (s. $3 \mathrm{H}) .7 .16$ (d. $J=8.1 \mathrm{~Hz} .2 \mathrm{H}) .7 .28$ (d. $J$ $=8.1 \mathrm{~Hz} \cdot 2 \mathrm{H}) .7 .80(\mathrm{~s} .1 \mathrm{H})$.

Compound 4: colorless oil: 86\%: IR (neat) 3294. 2952. $2258.1730 .1279 \mathrm{~cm}^{-1} ;{ }^{1} \mathrm{H} \mathrm{NMR}(\mathrm{CDCl}, 300 \mathrm{MHz}) \delta 2.07$ (t. $J=2.7 \mathrm{~Hz} .1 \mathrm{H}$ ). 2.78 (dd. $J=16.8$ and $3.0 \mathrm{~Hz} .1 \mathrm{H}$ ). 2.98 (d. $J=16.8$ and $3.0 \mathrm{~Hz}, 1 \mathrm{H}$ ) 3.67 (s. $3 \mathrm{H}), 3.68(\mathrm{~s} .6 \mathrm{H}), 5.04$ (s. $1 \mathrm{H}$ ). 6.19 (s. $1 \mathrm{H}) .6 .46$ (s. $1 \mathrm{H}) .7 .22$ (d. $J=8.7 \mathrm{~Hz} .2 \mathrm{H}$ ). $7.30(\mathrm{~d} J=8.7 \mathrm{~Hz}, 2 \mathrm{H})$

Typical procedure for the radical cyclization of 2a: A misture of 2 a (172 mg. $0.5 \mathrm{mmol}$ ). AIBN (2 $\mathrm{mg} .0 .01 \mathrm{mmol}$ ). and $n-\mathrm{Bu} 3 \mathrm{~S}_{3} \mathrm{SnH}$ ( $\left.160 \mathrm{mg} .0 .55 \mathrm{mmol}\right)$ in benzene $(3 \mathrm{~mL})$ was heated to reflux for $l \mathrm{~h}$. The reaction mixture was diluted with ether and a few drops of $c-\mathrm{HCl}$ solution was added and stirred for $l \mathrm{~h}$. After the usual aqueous extractive workup with ether and column chromatographic purification process (hexanes/EtOAc. $7: 3$ ) we obtained 3a (156 mg. 95\%) as colorless oil. Other compounds $3 \mathrm{~b}-\mathrm{f}$ were synthesized analogously and the spectroscopic data of 3a-f are as follows.

Compound 3a: colorless oil: 95\%: IR (neat) 2952.1732. 1496. $1435.1201 \mathrm{~cm}^{-1} ;{ }^{1} \mathrm{H} \mathrm{NMR}\left(\mathrm{CDCl}_{3}, 300 \mathrm{MHz}\right) \delta 2.55$ $($ d. $J=14.4 \mathrm{~Hz} . \mathrm{lH}), 2.66(\mathrm{~d} . J=16.5 \mathrm{~Hz}, \mathrm{lH}) .2 .68(\mathrm{~d} . J$ $=13.8 \mathrm{~Hz}, 1 \mathrm{H}) .2 .77(\mathrm{~d} . J=14.4 \mathrm{~Hz} .1 \mathrm{H}) .3 .12(\mathrm{~d} . J=16.5$ Hz. $1 \mathrm{H}) .3 .36(\mathrm{~d} . J=13.8 \mathrm{~Hz}, \mathrm{lH}) .3 .56$ (s. $3 \mathrm{H}) .3 .60$ (s. $3 \mathrm{H})$. 3.63 (s. $3 \mathrm{H}$ ). 5.13 (t. $J=2.1 \mathrm{~Hz} .1 \mathrm{H}$ ). 5.24 (t. $J=2.1 \mathrm{~Hz}$. lH) $7.07-7.20(\mathrm{~m} .5 \mathrm{H}):{ }^{13} \mathrm{C}$ NMR $\left(\mathrm{CDCl}_{3} .75 \mathrm{MHz}\right) \delta$ $40.06,40.79 .44 .17,52.16,52.56 .52 .68,56.90,57.62$. $110.27,126.55$. 128.10. 129.58, 137.31. 150.31, 171.30. $171.83,173.75$

Compound 3b: colorless oil: 90\%: IR (neat) 2952. 1736. 1493. 1435. 1263. $1201 \mathrm{~cm}^{-1}$, ${ }^{1} \mathrm{H} \mathrm{NMR}\left(\mathrm{CDCl}_{3}, 300 \mathrm{MHz}\right) \delta$ $2.60($ d. $J=14.4 \mathrm{~Hz} . \mathrm{IH}) .2 .75($ d. $J=13.8 \mathrm{~Hz}, \mathrm{lH}) .2 .76($ d. $J=16.2 \mathrm{~Hz}, \mathrm{lH}) .2 .82(\mathrm{~d}, J=14.4 \mathrm{~Hz} . \mathrm{lH}), 3.22(\mathrm{~d} . J=16.2$ Hz. $1 \mathrm{H}) .3 .40(\mathrm{~d} . J=13.8 \mathrm{~Hz}, 1 \mathrm{H}) .3 .65$ (s. $3 \mathrm{H}) .3 .71$ (s. $3 \mathrm{H})$.
3.73 (s. $3 \mathrm{H}$ ). 5.22 (t. $J=2.1 \mathrm{~Hz} .1 \mathrm{H}$ ). 5.29 (t. $J=2.1 \mathrm{~Hz}$. $1 \mathrm{H}) .7 .12$ (d. $J=8.4 \mathrm{~Hz}, 2 \mathrm{H}), 7.22(\mathrm{~d} . J=8.4 \mathrm{~Hz}, 2 \mathrm{H}):{ }^{12} \mathrm{C}$ NMR $\left(\mathrm{CDCl}_{3}, 75 \mathrm{MHz}\right) \delta 40.14 .40 .96 .43 .47 .52 .44 .52 .80$. $52.92 .56 .94,57.81,110.61 .128 .40,131.16,132.65 .135 .97$. $150.25,171.39 .172 .01,173.78$

Compound 3c: colorless oil: 95\%: IR (neat) 2981. 2954 1732. $1601,1583.1261,1194 \mathrm{~cm}^{-1}$ : ${ }^{1} \mathrm{H}$ NMR (CDCl. 300 MHz) $\delta 1.20-1.26(\mathrm{~m}, 6 \mathrm{H}), 2.64(\mathrm{~d} . J=14.7 \mathrm{~Hz}, \mathrm{lH}) .2 .74$ (d. $J=13.5 \mathrm{~Hz}, 1 \mathrm{H}), 2.77(\mathrm{~d}, J=16.5 \mathrm{~Hz}, 1 \mathrm{H}), 2.86(\mathrm{~d} . J=$ $14.7 \mathrm{~Hz} .1 \mathrm{H}) .3 .18(\mathrm{~d} . J=16.5 \mathrm{~Hz}, 1 \mathrm{H}) .3 .42(\mathrm{~d} . J=13.5 \mathrm{~Hz}$. 1H). $4.08-4.24(\mathrm{~m}, 4 \mathrm{H}) .5 .20(\mathrm{t} . J=2.1 \mathrm{~Hz}, \mathrm{lH}) .5 .30(\mathrm{t} . J=$ $2.1 \mathrm{~Hz}, 1 \mathrm{H}) .6 .74-6.79(\mathrm{~m}, 3 \mathrm{H}), 7.14-7.20(\mathrm{~m}, 1 \mathrm{H}):{ }^{13} \mathrm{C}$ NMR $\left(\mathrm{CDCl}_{3}, 75 \mathrm{MHz}\right) \delta 13.91 .39 .82,40.26,44.26 .52 .24 .54 .99$, $56.97,57.82,61.45,61.58,110.15,111.92,115.51,122.15$. 129.10. 139.07. 150.73, 159.36, 171.01. 171.58, 173.93.

Compound 3d: colorless oil: 97\%: IR (neat) 2952, 1732. 1612. $1512,1435,1252 \mathrm{~cm}^{-1}$. ${ }^{1} \mathrm{H}$ NMR $\left(\mathrm{CDCl}_{3} .300 \mathrm{MHz}\right) \delta$ $2.62(\mathrm{~d}, J=14.1 \mathrm{~Hz}, \mathrm{lH}) .2 .7 \mathrm{l}(\mathrm{d}, J=13.8 \mathrm{~Hz} . \mathrm{lH}), 2.74(\mathrm{~d}$, $J=16.5 \mathrm{~Hz}, 1 \mathrm{H}$ ). 2.84 (d. $J=14.1 \mathrm{~Hz}, 1 \mathrm{H}$ ). $3.20($ d. $J=16.5$ Hz. $1 \mathrm{H}$ ). 3.37 (d. $J=13.8$ Hz. 1H). 3.65 (s. $3 \mathrm{H}$ ). 3.70 (s. $3 \mathrm{H}$ ). 3.73 (s. $3 \mathrm{H}) .3 .77$ (s. $3 \mathrm{H}), 5.20(\mathrm{t} . J=2.1 \mathrm{~Hz}, 1 \mathrm{H}) .5 .30(\mathrm{t} . J$ $=2.1 \mathrm{~Hz} .1 \mathrm{H}) .6 .80(\mathrm{~d} . J=8.7 \mathrm{~Hz}, 2 \mathrm{H}) .7 .10(\mathrm{~d} . J=8.7 \mathrm{~Hz}$. $2 \mathrm{H}):{ }^{13} \mathrm{C}$ NMR $\left(\mathrm{CDCl}_{3} .75 \mathrm{MHz}\right) \delta 40.14,40.93 .43 .47$. $52.23,52.65,52.77,55.03,57.17,57.70,110.30$. 113.57. $129.41,130.68$. 150.32, 158.32, 171.43. 171.96, 173.93.

Compound 3e: colorless oil: 90\%: IR (neat) 2954, 2245. 1739. $1493,1246 \mathrm{~cm}^{-1},{ }^{1} \mathrm{H}$ NMR (CDCl $\left.3.300 \mathrm{MHz}\right) \delta 1.33$ (t. $J=7.2 \mathrm{~Hz}, 3 \mathrm{H}), 2.42(\mathrm{~d} . J=14.1 \mathrm{~Hz}, 1 \mathrm{H}) .2 .82(\mathrm{~d} . J=$ $13.8 \mathrm{~Hz}, 1 \mathrm{H}) .2 .94(\mathrm{~d} . J=15.3 \mathrm{~Hz} .1 \mathrm{H}) .2 .97-3.00(\mathrm{~m}, 2 \mathrm{H})$. 3.42 (d. $J=13.8 \mathrm{~Hz}, 1 \mathrm{H}$ ). 3.75 (s. $3 \mathrm{H}) .4 .27$ (q. $J=7.2 \mathrm{~Hz}$. $2 \mathrm{H}) .5 .31(\mathrm{t} . J=1.8 \mathrm{~Hz} .1 \mathrm{H}) .5 .45(\mathrm{t} . J=1.8 \mathrm{~Hz} .1 \mathrm{H}) .7 .09$ (d. $J=8.4 \mathrm{~Hz}, 2 \mathrm{H}), 7.25$ (d. $J=8.4 \mathrm{~Hz}, 2 \mathrm{H}):{ }^{13} \mathrm{C}$ NMR $\left(\mathrm{CDCl}_{3}, 75 \mathrm{MHz}\right) \delta 13.90,42.01 .43 .91 .43 .99,45.84 .52 .72$. $56.85,63.29$. 112.54, 119.26, 128.57, 131.08, 132.98 . $135.18,147.88 .168 .42,172.95$.

Compound 3f: colorless oil: 93\%: IR (neat) 2952. 1718. 1435. $1254,1198 \mathrm{~cm}^{-1}:{ }^{1} \mathrm{H}$ NMR $\left(\mathrm{CDCl}_{3}, 300 \mathrm{MHz}\right) \delta 2.17$ (s. $3 \mathrm{H}) .2 .31$ (s. $3 \mathrm{H}) .2 .60(\mathrm{~d} . J=14.4 \mathrm{~Hz} .1 \mathrm{H}) .2 .65(\mathrm{~d} . J=$ $16.5 \mathrm{~Hz} \cdot 1 \mathrm{H}) .2 .71(\mathrm{~d} . J=13.8 \mathrm{~Hz}, 1 \mathrm{H}), 2.75(\mathrm{~d} . J=14.4 \mathrm{~Hz}$. $1 \mathrm{H}), 3.22(\mathrm{~d} . J=16.5 \mathrm{~Hz}, 1 \mathrm{H}) .3 .41(\mathrm{~d} . J=13.8 \mathrm{~Hz}, 1 \mathrm{H})$. 3.64 (s. $3 \mathrm{H}) .3 .73$ (s. $3 \mathrm{H}), 5.20(\mathrm{t} . J=2.1 \mathrm{~Hz}, \mathrm{lH}) .5 .30$ (t. $J$ $=2.1 \mathrm{~Hz}, 1 \mathrm{H}), 7.03-7.09(\mathrm{~m}, 4 \mathrm{H}):{ }^{13} \mathrm{C}$ NMR $\left(\mathrm{CDCl}_{3}, 75\right.$ MHz) $\delta 21.00 .25 .99 .39 .14,39.39,44.14$. 52.23. 52.81, $57.16,64.29 .110 .26 .129 .02 .129 .53,134.32,136.28$, $150.46,172.71,173.98,202.63$

Synthesis of 5: To a stirred solution of $3 \mathrm{a}$ (104 mg. 0.3 munol) in benzene $\left(3 \mathrm{~mL}\right.$ ) was added $\mathrm{H}_{2} \mathrm{SO}_{4}(88 \mathrm{mg}, 0.9$ mumol) at $0{ }^{\circ} \mathrm{C}$ and stirred the reaction mixture at room temperature for $\mathrm{l} \mathrm{h}$. After the usual aqueous extractive workup with ether and column chromatographic purification process (hexanes/EtOAc. $7: 3$ ) we obtained 5 ( $89 \mathrm{mg} .86 \%$ ) as colorless oil.

Compound 5: colorless oil: $86 \%$ : IR (neat) 2954.1734 . $1435.1263 \mathrm{~cm}^{-1}$ : ${ }^{1} \mathrm{H}$ NMR $\left(\mathrm{CDCl}_{3} .300 \mathrm{MHz}\right) \delta 1.81(\mathrm{~d} . J=$ $1.5 \mathrm{~Hz}, 3 \mathrm{H}), 2.60(\mathrm{~d} . J=3.0 \mathrm{~Hz}, \mathrm{lH}) .2 .63(\mathrm{~d} . J=3.0 \mathrm{~Hz}$. $1 \mathrm{H}) .2 .81$ (d. $J=14.0 \mathrm{~Hz}, 1 \mathrm{H}) .3 .37(\mathrm{~d} . J=14.0 \mathrm{~Hz} .1 \mathrm{H})$, 3.58 (s. $3 \mathrm{H}$ ). 3.61 (s. $3 \mathrm{H}$ ). 3.62 (s. $3 \mathrm{H}$ ). 5.54 (t. $J=1.5 \mathrm{~Hz}$. 
1H), 7.10-7.20 (m. $5 \mathrm{H}) ;{ }^{13} \mathrm{C}$ NMR $\left(\mathrm{CDCl}_{3} .75 \mathrm{MHz}\right) \delta$ 13.92, 38.92. 41.28, 52.03, 52.66, 52.89, 62.22, 64.31. $126.09,126.63,128.26,129.85,137.23,146.30,171.26$. 171.27. 173.76: LCMS $346\left(\mathrm{M}^{-}\right)$.

Acknowledgments. This study was financially supported by Chomnam National University (2005). Spectroscopic data was obtained from the Korea Basic Science Institute, Gwangiu branch.

\section{References and Notes}

1. For the synthesis and biological activities of cyclopentanecontaining compounds. see: (a) Ye. Q: Grunewald. G. L.J. Med. Chem. 1989, 32, 478. (b) Scott. K. R. Moore. J. A.: Zalucky. T B.: Nicholson. J. M.: Lee, J. A. M.: Hinko, C. N. J. Med. Chem. 1985, 28. 413. (c) Amori, L.: Costantino. G: Marinozzi, M: Pellicciari. R: Gasparini, F.: Flor, P. I.: Kulun, R: Vranesic, I. Bioorg. Hed Chem. Lett. 2000. 10. 1447. (d) Greene. A. E.: Luche. M.-J.: Depres. J.-P. J. An Chem. Soc. 1983. 105. 2435. (e) Depres. J.-P.: Greene. A. E. J. Org. Chem. 1984. 49.928.

2. For the synthesis of exo-methylenecyclopentane ring by radical cyclization or evelization of enyines involving $\mathrm{Rh}$. $\mathrm{Pd}$ or $\mathrm{All}$ catalyst. see: (a) Gomez, A. M. Company. M. D.: Uriel, C.: Valverde. S.: Lopez. J. C. Tetrohedron Lett. 2002. 13. 4997. (b) Curran. D. P.: Chen. M.-H.: Spletzer. E.: Seong. C. M.: Chang. C.T. J.Am. Chem Soc. 1989. H1. 8872 (c) Miura. T.: Shimada. M: Murakani. M. J. Am. Chem. Soc, 2005, 127, 1094. (d) Aggarwal.
V. K. Butters, M.: Davies, P. W. Chem. Commm 2003. 1046. (e) Oh. C. H.: Sun1g. H. R.: Park. S. T.: Aht1. K. H. J. Org. Chent. 2002. 67. 7155. (f) Oh. C. H.: Tung. H. H.: Sung. H. R.: Kim. I. D. Tetrahedron 2001. 57. 1723. (g) Oh. C. H.: . Tung. H. H. Tetrahedron Lett. 1999, t0, 1535. (h) Kennedy-Smith. J. J.: Staben. S. T. Toste, F. D. J. Am. Chem. Soc. 2004, 126. 4526.

3. Lee. K. Y.: Na. J. E; Lee, J. Y: Kim. J. N. Bull. Korean Chem Soc. 2004. 25. 1280 .

4. (a) Gowrisankar. S.: Lee. K. Y.: Kim. J. N. Tetrahed-on Lett. 2005. 46. 4859. (b) Shanmugam. P.: Rajasingh. P. Tetrohedron Lett. 2005. 46. 3369. (c) Shanmugam. P. Rajasingh. P. Tetohledron 2004, 60.9283. (d). Shanmugam, P; Rajasingh. P. Synlett 2005. 939. (e) For the regio- and stereoselective synthesis of methyl 5methylenetetrahydropyran-3-carboxylates from Baylis-Hillman adducts via allyltributylstannane-mediated radical cyclization. please see: Gowrisankar. S.: Lee. K. Y.: Kim. T. H.: Kim. J. N. Tetrahedron Lett. 2006. 47.5785.

5. For the introduction of malonate derivatives to Baylis-Hillman adducts, see: (a) Lee, M. J.; Park, D. Y: Lee, K. Y.: Kim, J. N. Tetrahedron Lett. 2006. 47. 1833. (b) Im. Y. T.: Lee. C. G.: Kim. H. R.: Kim. J. N. Tetrohdron Lett. 2003. H. 2987. (c) Im. Y. J.: Lee. K. Y: Kim. T. H.: Kim. T. N. Tetrohdron Leth. 2002. 43. 4675. (d) Im. Y. J.: Kim, J. M; Kim. J. N. Bull. Koman Chem. So. 2002. 23. 1361 .

6. For the references on regioselective introduction of nucleophiles at the secondary position of the Baylis-Hillman adducts by using the DABCO salt concept. see: (a) Kim. J. N.: Kiml. T. M.: Lee. K. Y.: Gowrisankar. S. Bull. Korean Chent Soc. 2004. 25. 1733. (b) Lee. K. Y; Gowrisankar, S.; Kim. J. N. Bull. Korean Chem. Soc. 2005, 26, 1481 and further references citied therein 